\title{
A new numerical method for DEM-block and particle model
}

\author{
Shihai Li*, Manhong Zhao, Yuannian Wang, Ying Rao \\ Institute of Mechanics, Chinese Academy of Sciences, Beijing 100080, China
}

\begin{abstract}
As pointed out in Jing and Hudson's 2002 review paper on numerical methods in rock mechanics, the distinct element method (DEM) is one of the important numerical methods for simulating the mechanical properties of rock masses. Many researchers have paid attention to block deformation and failure in DEM. The updated DEM can be classified into two types: one is the particle model; and the other is the block model. This paper describes the development of a new numerical model, the block and particle model (BPM), to simulate both continuous and discontinuous deformations of rock masses. Both elastic deformation and failure of blocks can be described in this model.

The model assumes that a rock mass consists of blocks and each block is formed by particles arranged in a specific way (see Fig. 1), thus formulating a combination of a block model and a particle model. In this model, blocks and particles are connected by springs. Between blocks, there are three springs for adjacent particles: one is a uniaxial spring and the other two are transverse springs. One block has 25 particles. One of the particles is in the center of the cube and the other 24 particles lie in the midpoint between the face center and the four corner points. Spring groups connect these particles.

This paper also describes the development of the forces in a block and between blocks and their spring stiffness. The rigidity coefficient of springs between blocks generates the mechanical characteristics of joints in the rock mass. They can be obtained by material tests or wave methods by means of the Goodman model. The rigidity coefficient of springs between particles depends on the characteristics of deformation and failure of a block. The block is cubic and uniform deformation is studied in this model. We assume that the displacement of each particle in a cube block is equal to the displacement of the same position point of the elastic block under the same and known loading; thus, nine equilibrium equations are obtained for each particle. Solving these equations, nine rigidity coefficients can be determined. These coefficients are the function for elastic modulus and Poisson's ratio. Furthermore, a new failure criterion is suggested for the block failure.

This model is verified by an example of rock masses under uniaxial loading. It is shown that numerical results agree well with theoretical ones and laboratory tests, not only for the deformation process but also for the failure process.
\end{abstract}

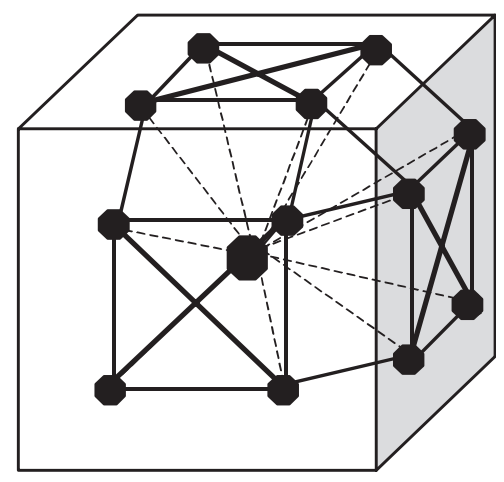

Fig. 1. Springs and particles in a block.

*Corresponding author. Tel.: + 86-10-62573764.

E-mail address: shli@imech.ac.cn (S. Li).

For full length paper see CD-ROM attached. 